\title{
Isolation, Purification, and Characterization of Ginger-derived Nanoparticles (GDNPs) from Ginger, Rhizome of Zingiber officinale \\ Junsik Sung ${ }^{1, *}$, Chunhua Yang ${ }^{1}$, Emilie Viennois ${ }^{1}$, Mingzhen Zhang ${ }^{1}$ and Didier Merlin ${ }^{1,2}$
}

${ }^{1}$ Institute for Biomedical Science, Center for Diagnostics and Therapeutics, Georgia State University, Atlanta, GA, USA; ${ }^{2}$ Atlanta Veterans Affairs Medical Center, Decatur, GA, USA

*For correspondence: jsung9@gsu.edu

[Abstract] Factors implicated in the pathophysiology of intestinal inflammation include defects in intestinal epithelial barrier function, abnormal immune responses, and activities of the gut microbiota. Current agents used to treat human Inflammatory Bowels Disease (IBD), chronic inflammation of digestive tract, have serious side effects. In addition, most of these treatments target the damaging factors while not providing pro-healing factors that repair the damaged intestine. Here we provide a method to isolate, purify and characterize a specific population from ginger (ginger-derived nanoparticles: GDNPs 2) with anti-inflammatory activities. GDNPs 2 as a drug vehicle are a novel natural, nontoxic delivery system, which target the inflamed intestinal mucosa, blocks damaging factors while promoting pro-healing factors and could easily be developed for large-scale production aimed at the treatment of IBD.

Keywords: Ginger, Sucrose gradient ultracentrifuge, Nanoparticles, Ginger-derived nanoparticles, GDNPs 2, Natural drug delivery system, Inflammatory Bowels Disease, IBD

[Background] Efforts to develop new drug-based therapeutic approaches against Intestinal Bowel Disease (IBD) must overcome several challenges, including issues of delivery, potential off-target effects, safety, toxicity, large-scale production costs, and tissue specificity (Chen et al., 2017). We and other groups have recently demonstrated that artificially synthesized nanoparticles may be used to target low doses of drugs (e.g., siRNAs, proteins and peptides) to specific cell types or tissues (Xiao et al., 2017). However, the nanoparticles synthesized to date have two major limitations: i) each constituent of the synthesized nanoparticles must be examined for potential in vivo toxicity before clinical application; and ii) the production scale is limited. The use of nanoparticles derived from natural sources may overcome these limitations of synthetic nanoparticles. Here, we provide a safe and cost-effective sucrose gradient ultracentrifuge method to isolate, purify and characterize a specific population of nanoparticles from ginger (ginger-derived nanoparticles: GDNPs 2) with anti-inflammatory activities. GDNPs 2 enable encapsulation of various types of therapeutic agents such as DNA, RNA and antibodies. Loading therapeutic agents in ginger-derived nanolipids can be found in a separate publication (Zhang et al., 2017). GDNPs 2 represents a novel natural, nontoxic delivery system that targets the inflamed intestinal mucosa, blocks damaging factors while promoting healing factors and may easily be developed for large-scale production aimed at the treatment of IBD. 


\section{Materials and Reagents}

1. Pipette tips 0.1-10 $\mu \mathrm{l}, 1-200 \mu \mathrm{l}, 100-1,000 \mu \mathrm{l}$ (Sorenson Bioscience, catalog numbers: 70600 , $70520,70540)$

2. Pipettes $0.5-10 \mu \mathrm{l}, 10-100 \mu \mathrm{l}$ and 100-1,000 $\mu \mathrm{l}$ (Eppendorf, catalog number: 13-684-251)

3. Thin wall, Ultra clear $13.2 \mathrm{ml}, 14 \times 89 \mathrm{~mm}$ ultracentrifuge tubes (Beckman Coulter, catalog number: 344059)

4. Thick wall, $38 \mathrm{ml}, 25 \times 89 \mathrm{~mm}$ centrifuge tubes polycarbonate (Beckman Coulter, catalog number: 355631)

5. Thick wall, $70 \mathrm{ml}, 38 \times 102 \mathrm{~mm}$ centrifuge bottles polycarbonate (Beckman Coulter, catalog number: 355655 )

6. Eppendorf Safe-Lock Tubes, $1.5 \mathrm{ml}$ (Eppendorf, catalog number: 022363204)

7. $50 \mathrm{ml}$ conical tubes (Denville Scientific, catalog number: C1062-P)

8. Formvar ${ }^{\circledR}$-coated copper grids (Electron Microscopy Sciences, catalog number: FCF300-CU$\mathrm{SC})$

9. Filter paper (VWR, catalog number: 28313-068)

10. Sterile cell strainer $40 \mu \mathrm{M}$ (Fisher Scientific, catalog number: 22-363-547)

11. Capillary cell \& plastic cap (Malvern, catalog number: DTS 1070)

12. Protein quantification assay kit (Bio-Rad, catalog number: 5000002)

13. Phosphate-buffered Saline (Corning, catalog number: 21-040-CV)

14. Simulated intestinal fluids (RICCA Chemical, catalog number: 7109-16)

15. Simulated gastric fluids (RICCA Chemical, catalog number: 7108-16)

16. Uranyl acetate (Electron Microscopy Sciences, catalog number: 22400-4)

17. Mica sheet (Electron Microscopy Sciences, catalog number: 71855-15)

18. Sucrose (Calbiochem, catalog number: 8510) (see Recipes)

19. Sucrose gradient $(8,30,45,60 \%)$ (see Recipes)

20. $1 \%$ uranyl acetate solution (see Recipes)

\section{Equipment}

1. Juicer (Breville, model: BJE200XL)

2. Sorvall ST $16 \mathrm{R}$ centrifuge (Thermo Fisher Scientific, catalog number: 75004381)

3. Optima L-90K Ultracentrifuge (Beckman Coulter, catalog number: 969349)

4. Rotors:

a. J-Lite Series JLA 16.250 16,000 RPM (Beckman Coulter, Serial number O8U 3761)

b. Type $45 \mathrm{Ti}$ 45,000 RPM (Beckman Coulter, Serial number 99E.2785)

c. SW 32 Ti 32,000 RPM (Beckman Coulter, Serial number 11U 2963)

5. Zetasizer (Malvern, model: Nano-ZS90)

6. Particle size analyzer (Brookhaven Instrument Corps, model: 90PLUS) 
7. Atomic force microscopy instrument (Seiko Instrument Inc., model: SPA400)

8. Transmission electron microscope (Carl Zeiss, model: LEO 906E)

9. Milli-Q advantage A10 water purification system (Millipore-sigma, catalog number: $\mathrm{C} 10117$ )

10. Semi-micro cuvettes, clear $1.5 \mathrm{ml}$ (Sigma, catalog number: BR759115)

11. Ultrasonic bath (Branson, catalog number: $3510 \mathrm{MTH}$ )

12. Fluorometric microplate reader (Biotek, model: Synergy 2)

13. $\mathrm{pH}$ meter (Sper Scientific, catalog number: 860031)

14. $-80{ }^{\circ} \mathrm{C}$ freezer (So-Low, model: PV85-21)

\section{Procedure}

A. Isolation (see Note 1) (Figure 1)

Note: Approximate amounts of GDNPs 2 acquired from $1 \mathrm{~kg}$ of ginger is $50-100 \mathrm{ng} / \mathrm{ml}$.

1. Wash $1 \mathrm{~kg}$ of roughly peeled ginger thoroughly with tap water.

2. Grind sliced ginger with blender to obtain juice.

3. Centrifuge approximately $550 \mathrm{ml}$ of the ginger juice at $3,000 \times \mathrm{g}$ for $20 \mathrm{~min}$ at RT.

4. Centrifuge again at $10,000 \times g$ for $2 \mathrm{~h}$ at RT to remove large fibers.

5. Collect the supernatant and equally distribute $(\sim 45 \mathrm{ml})$ into twelve $70 \mathrm{ml}$ capacity tubes.

6. Ultracentrifuge the collected supernatant at $4{ }^{\circ} \mathrm{C}, 150,000 \times \mathrm{g}$ for $2 \mathrm{~h}$ (Use Type $45 \mathrm{Ti} 45,000$ RPM). Ultracentrigfuge 6 tubes two times.

7. Remove supernatant and suspend the pellet in each tube in $5 \mathrm{ml}$ PBS through ultrasonic dispersion. After proper pipetting, all the nanoparticle pellets should be well suspended in PSC solution. If insoluble pellet exists, a cell strainer can be used to remove the insoluble pellet before loading to the gradient centrifugation.
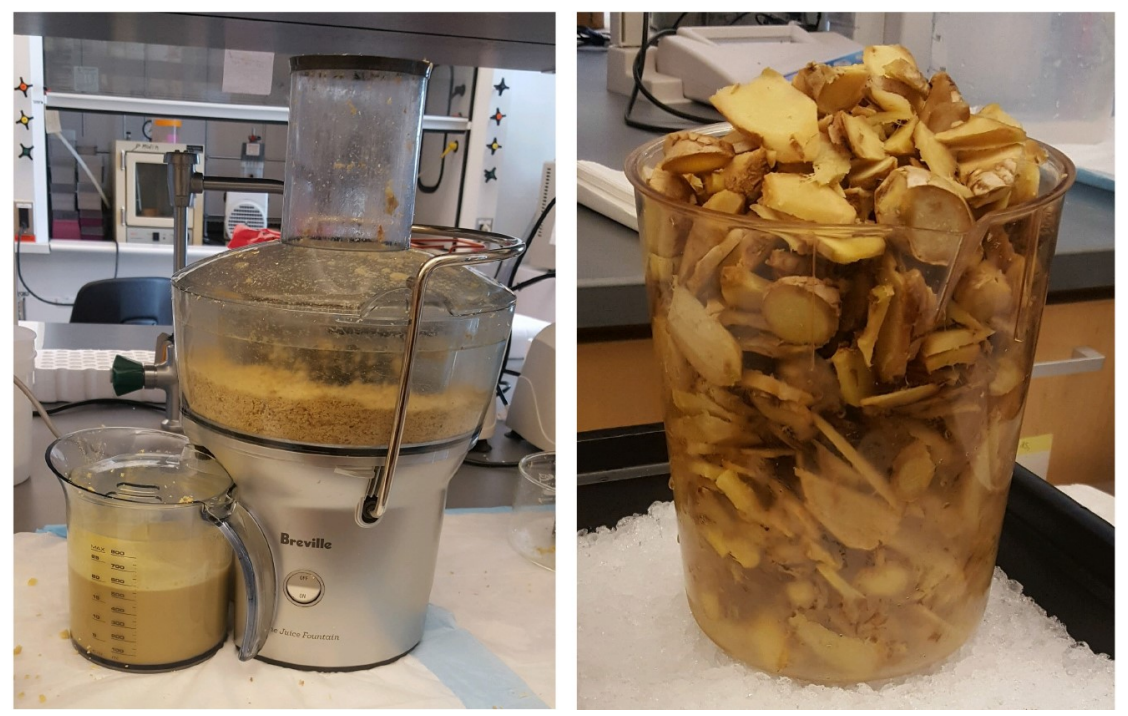

Figure 1. Ginger juice extraction. Sliced ginger is squeezed in the juicer to obtain juice for nanoparticle isolation and purification. 


\section{B. Purification}

1. Prepare twelve $38.5 \mathrm{ml}$ capacity tubes filled with $10 \mathrm{ml}$ of each sucrose solution in the order of $8 \%, 30 \%$ and $45 \%$ from top to bottom.

2. Transfer $5 \mathrm{ml}$ of the PBS suspended pellet from each tube to discontinuous sucrose gradient $\left(8 \%, 30 \%, 45 \%\right.$ [gram/volume]) in the 12 tubes and ultracentrifuge with SW 32 Ti rotor at $4{ }^{\circ} \mathrm{C}$, $150,000 \times g$ for $2 \mathrm{~h}$ (see Recipe 1). Ultracentrigfuge 6 tubes two times.

3. Collect $\sim 2 \mathrm{ml}$ of band 2 and $\sim 0.6 \mathrm{ml}$ of band 1 respectively from $8 / 30 \%$ and $30 / 45 \%$ interfaces by gently dipping pipette tips into the sucrose gradient solution (see Data analysis).

4. Ultracentrifuge the collected bands at $4{ }^{\circ} \mathrm{C}, 100,000 \times g$ for $45 \mathrm{~min}$ (use Type $45 \mathrm{Ti} 30,000 \mathrm{RPM}$ ), remove the supernatant.

5. Wash the pellet with $1 \mathrm{ml}$ PBS to remove the remaining sucrose and then suspend the pellet in $1 \mathrm{ml}$ PBS through ultrasonic dispersion.

6. Measure the concentration of obtained GDNPs $(0.25 \sim 1.25 \mathrm{mg} / \mathrm{ml})$ using protein quantification assay kit (follow the manufacturer's manual) and microplate reader at absorbance of $750 \mathrm{~nm}$.

Note: Proteins contained by GDNPs were used to quantitate the GDNPs. The assay is based on the reaction of protein with an alkaline copper tartrate solution and Folin reagent (DC Protein Assay Instruction Manual). Proteins effect a reduction of the Folin reagent by loss of 1, 2, or 3 oxygen atoms, thereby producing one or more of several possible reduced species which have a characteristic blue color with maximum absorbance at $750 \mathrm{~nm}$. Color development is primarily due to the amino acid tyrosine and tryptophan groups.

7. For short term storage (less than 3 months), GDNPs can be suspended in PBS and stored at $80^{\circ} \mathrm{C}$. Long term stability has not been tested (Only Band 2 for Characterization; Discard Band 1).

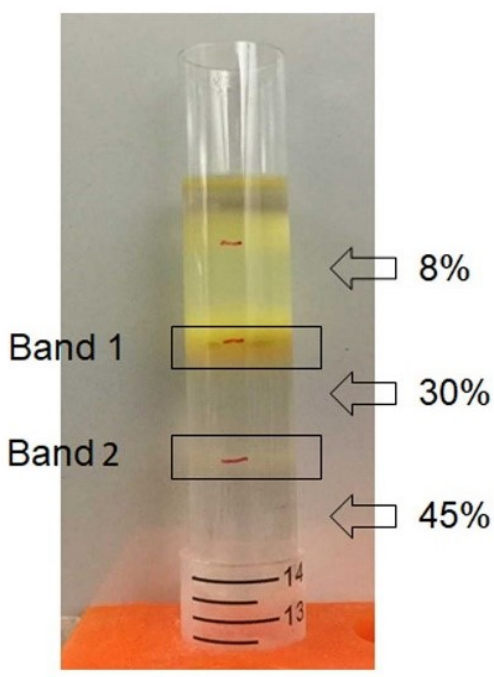

Figure 2. Sucrose gradient ultracentrifugation. GDNPs were added to $8,30,45 \%$ of sucrose gradient and ultracentrifuged for purification. 


\section{Characterization (GDNPs 2)}

1. Test the nanoparticle for its in vitro stability (see Note 6)

Note: Measure particle size and zeta potential before and after incubation in SGF/IGF for comparison.

a. Add $1 \mathrm{ml}$ of nanoparticles $(1 \mathrm{mg} / \mathrm{ml})$ to $9 \mathrm{ml}$ of simulated gastric fluid (SGF) and simulate intestinal fluid (SIF) in a separate tube.

b. Mix thoroughly by inverting tubes.

c. Dilute $0.2 \mathrm{ml}$ of mixture into $1.8 \mathrm{ml}$ PBS.

d. Determine the particle size and zeta potential before incubation (follow Steps C2 and C3 below).

e. Incubate the mixture in $37^{\circ} \mathrm{C}$ water bath for $30 \mathrm{~min}$.

f. Determine the particle size and zeta potential after incubation.

2. Measure particle size (Instruction for Steps $C 1 d$ and $C 1 f$, see Data analysis, Figure 2)

a. Dilute $0.2 \mathrm{ml}$ of mixture into $1.8 \mathrm{ml}$ PBS.

b. Carefully add all of the diluted mixture to a cuvette without bubbles.

c. Insert the cuvette to the particle analyzer chamber.

d. Determine the size of particles.

3. Measure zeta potential (Instruction for Steps C1d and C1f, see Data analysis, Figure 1)
a. Carefully add $0.8 \mathrm{ml}$ of mixture to capillary cell.
b. Cover the capillary cell with plastic cap.
c. Insert the cell to the chamber of zeta potential analyzer.
d. Determine the numbers for the sample at neutral $\mathrm{pH}$.

4. Acquire transmission electron microscopy (TEM) image (Figure 5)
a. Deposit a drop of sample onto the surface of a formvar-coated grid.
b. Add $1 \%$ uranyl acetate on top of the sample and wait for $15 \mathrm{~s}$ (see Recipe 2).
c. Carefully absorb the sample and uranyl acetate with tissue without touch the surface.
d. Dry the sample at room temperature for $5 \mathrm{~min}$.
e. Scan the sample image with a transmission electron microscope.

5. Acquire atomic force microscopy (AFM) image (Figure 6)
a. Deposit $1 \mu \mathrm{l}$ of nanoparticle sample to mica sheet.
b. Dry the sample at room temperature.
c. Gently rinse the mica sheet with $2.5 \mu \mathrm{l}$ of distilled water three times.
d. Dry the sample at room temperature again.
e. Leave it until the sample become flat.
f. Scan the sample with area of about $4 \times 4 \mu \mathrm{m}$ and 2-50 $\mathrm{nm}$ in height.

\section{Data analysis}

Sucrose gradient ultracentrifuge methods showed two concentrated bands, $8 / 30 \%$ (band 1 ) and 
30/45\% (band 2). Photon correlation spectroscopy (PCS) was used to measure size distribution and zeta potential of the GDNPs. The average nanoparticle size of band 2 was approximately $\sim 115.8$ $\mathrm{nm}$ in radius (Figure 3). An approximate zeta potential value was $\sim 12 \mathrm{mV}$ at $\mathrm{pH} 6$ for band 2 (Figure 4). Transmission electron microscopy (TEM) and atomic force microscopy (AFM) analysis also confirmed successful isolation and characterization of stable GDNPs (Figures 5 and 6 ). In vitro stability test for GDNPs 2 confirmed the tolerance of the nanoparticle within harsh environment such as gastric and intestinal tract. Precisely measured particle size and zeta potential of GDNPs 2 ensure the capability to be used as stable drug carrier in human system. Also, TEM and AFM probed ability of GDNPs 2's electromechanical functionality which is critical for characterization for biological materials.

Size Distribution by Intensity

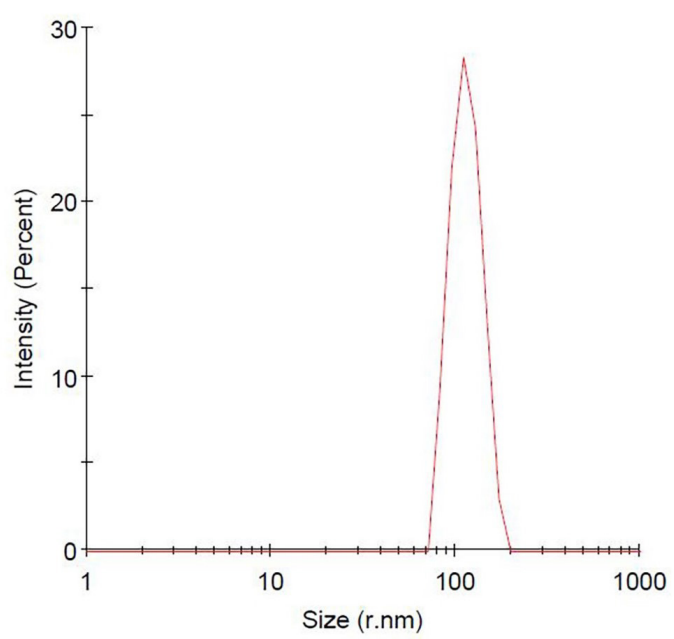

Figure 3. Measurement of band 2 ginger nanoparticle size. Approximate nanoparticle size was determined to be $\sim 115.8 \mathrm{~nm}$ in radius.

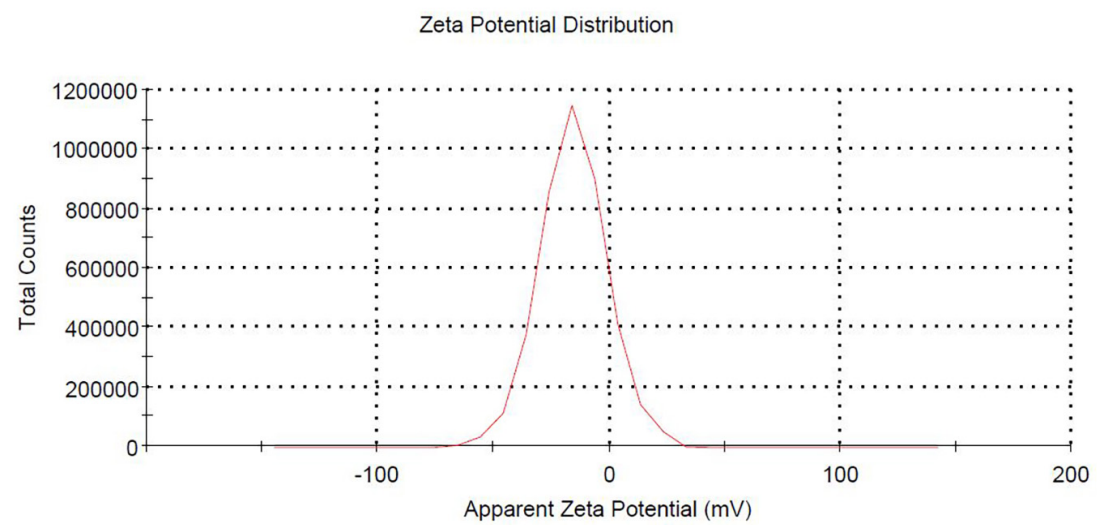

Figure 4. Measurement of zeta potential of band 2 ginger nanoparticle. Approximate zeta potential measured by Malvern zetasizer was $\sim-12 \mathrm{mV}$ at $\mathrm{pH} 6$. 


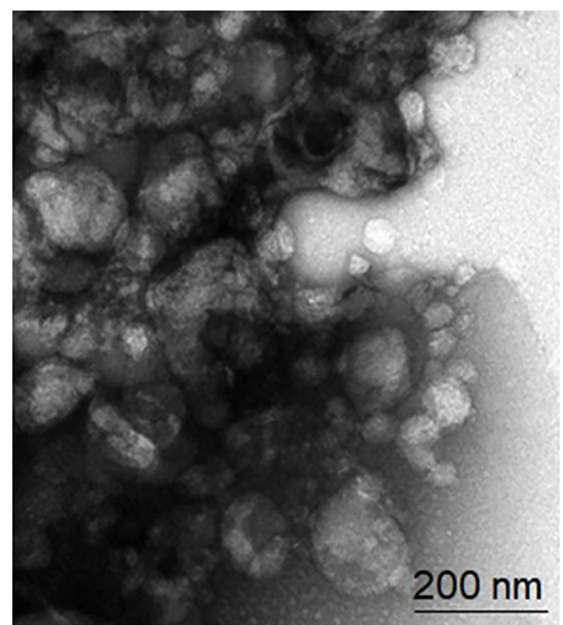

Figure 5. TEM (Transmission electron microscopy) image of GDNPs 2

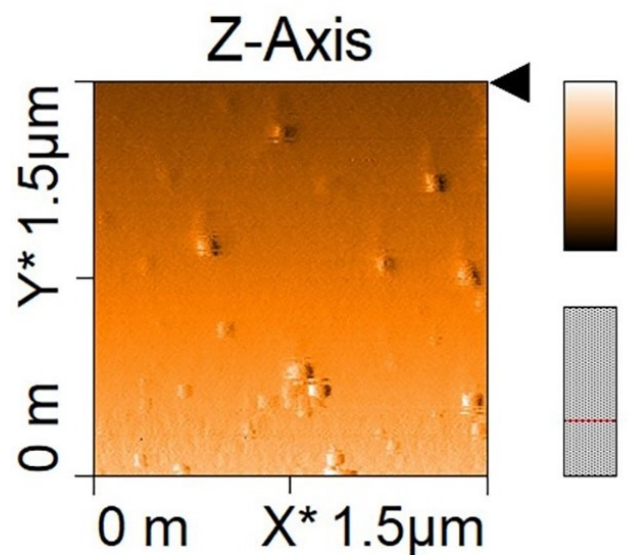

Figure 6. AFM (Atomic force microscopy) image of GDNPs 2

\section{$\underline{\text { Notes }}$}

1. Filtrate the sucrose solutions with $0.22 \mu \mathrm{m}$ membrane filter to eliminate small particles.

2. Pre-warm water bath to $37^{\circ} \mathrm{C}$.

3. Be sure to balance the particle size analyzer and zetasizer for at least $15 \mathrm{~min}$ before use.

4. Avoid generating air bubbles in cuvettes for size and zeta potential measurement.

\section{Recipes}

1. Sucrose gradient $(8,30,45 \%)$

a. Dissolve each $8,30,45 \mathrm{~g}$ sucrose powder in $70 \mathrm{ml}$ distilled water then make up the volume to $100 \mathrm{ml}$

b. Pipette each sucrose solution to make a gradient in the order from the bottom; $45 \%, 30 \%$,

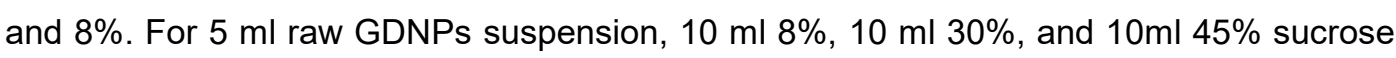
solution were used. Total $30 \mathrm{ml}$ of sucrose gradient is filled in the $38 \mathrm{ml}$ capacity tubes 
2. $1 \%$ uranyl acetate solution

a. Dissolve $4 \mathrm{~g}$ uranyl acetate powder into $100 \mathrm{ml}$ preheated $\left(50-60{ }^{\circ} \mathrm{C}\right)$ filtered water to prepare $4 \%$ uranyl acetate stock solution

b. Dilute $1 \mathrm{ml} 4 \%$ uranyl acetate stock solution into $3 \mathrm{ml}$ of filtered water

\section{Acknowledgments}

This work was supported by National Institute of Health of Diabetes and Digestive and Kidney (RO1DK-107739 \& RO1-DK-116306 to D.M) and the Department of Veterans Affairs (Merit Award BX002526 to D.M). D. Merlin is a recipient of a Senior Research Career Scientist Award from the Department of Veteran Affairs (BX004476). EV is a recipient of the Career Development Award from the Crohn's and Colitis Foundation. This protocol was adapted from published work by Zhang et al. (2016).

\section{Competing interests}

The authors declare no conflicts of interest within the work.

\section{References}

1. Chen, Q., Xiao, B. and Merlin, D. (2017). Nanotherapeutics for the treatment of inflammatory bowel disease. Expert Rev Gastroenterol Hepatol 11(6): 495-497.

2. Xiao, B., Ma, L. and Merlin, D. (2017). Nanoparticle-mediated co-delivery of chemotherapeutic agent and siRNA for combination cancer therapy. Expert Opin Drug Deliv 14(1): 65-73.

3. Zhang, M., Viennois, E., Prasad, M., Zhang, Y., Wang, L., Zhang, Z., Han, M. K., Xiao, B., Xu, C., Srinivasan, S. and Merlin, D. (2016). Edible ginger-derived nanoparticles: A novel therapeutic approach for the prevention and treatment of inflammatory bowel disease and colitis-associated cancer. Biomaterials 101: 321-340.

4. Zhang, M., Wang, X., Han, M. K., Collins, J. F. and Merlin, D. (2017). Oral administration of ginger-derived nanolipids loaded with siRNA as a novel approach for efficient siRNA drug delivery to treat ulcerative colitis. Nanomedicine (Lond) 12(16): 1927-1943. 